\title{
Questes
}

Revue pluridisciplinaire d'études médiévales

\section{La part de l'imaginaire chez Raoul Lefèvre : Jason à la cour de la reine Mirro}

\section{Natalia Bercea-Bocskai}

\section{(2) OpenEdition}

Édition électronique

URL : http://journals.openedition.org/questes/1534

DOI : 10.4000/questes.1534

ISSN : 2109-9472

Éditeur

Les Amis de Questes

Édition imprimée

Date de publication : 15 janvier 2008

Pagination : 81-95

ISSN : 2102-7188

\section{Référence électronique}

Natalia Bercea-Bocskai, « La part de l'imaginaire chez Raoul Lefèvre : Jason à la cour de la reine Mirro », Questes [En ligne], 13 | 2008, mis en ligne le 01 janvier 2014, consulté le 20 avril 2019. URL : http:// journals.openedition.org/questes/1534; DOI : 10.4000/questes.1534 


\title{
La part de l'imaginaire chez Raoul Lefèvre : Jason à la cour de la reine Mirro
}

\author{
Natalia BERCEA-BOCSKAI
}

Ouvrage entrepris vers 1460 et dédié à Philippe de Bourgogne, possible commanditaire, l'Histoire de Jason de Raoul Lefèvre se définit comme un récit qui se propose de rétablir toute la vérité sur la vie de l'Argonaute, conquérant légendaire de la toison d'or, et de réaffirmer son exemplarité. Jason, patron du fameux ordre bourguignon créé par le Grand Duc trente ans auparavant ${ }^{1}$, retrouve progressivement une « centralité narrative » qu'il avait perdue dans les écrits antiques : de "héros évincé », il redevient en cette fin de Moyen Âge l'actant principal des séquences de cette fable mythique ${ }^{2}$. Le prologue réunit en une mise en présence symbolique les trois figures-clés du contexte de création de l'œuvre : le clerc, le prince et le héros. Dans cette partie liminaire construite sur un franchissement des seuils narratifs, c'est Jason lui-même, révolté et attristé, qui s'adresse à l'écrivain :

\footnotetext{
${ }^{1}$ Voir Françoise De GRUBEn, Les Chapitres de la Toison d'or à l'époque bourguignonne (1430-1477), Louvain, Leuven University Press, 1997, p. 3-53; et le chapitre collectif « Histoire de l'Ordre de la Toison d'or », dans L'ordre de la Toison d'or, de Philippe le Bon à Philippe le Beau (1430-1505) : idéal ou reflet d'une société ?, dir. Pierre CocKSHAw, éd. Christiane VAN Den Bergen-Pantens, Bruxelles, Bibliothèque royale de Belgique, Turnhout, Brepols, 1996, p. 65-83. Sur cette référence problématique voir Georges Doutrepont, La Littérature française à la cour des ducs de Bourgogne, Paris, Honoré Champion, 1909, p. 147-171 et Lucie MARIGNAC, «Philippe le Bon et l'Ordre de la Toison d'or: les enjeux d'une référence mythique », Mythes et histoire, RAZO, Cahiers du Centre d'Études Médiévales de Nice 12, 1992, p. 87-112.

${ }^{2}$ Voir sur ce point l'étude de Paola-Maria FiLIPPI, «Réception du mythe de Médée au Moyen Âge ", dans La représentation de l'Antiquité au Moyen Age, Actes du Colloque de l'Université de Picardie, 26, 27 et 28 mars 1981, éd. par Danielle BusCHINGER et André CRÉPIN, Vienne, Verlag Karl M. Halosar, 1982, p. 91-101 : p. 92 et celle de Lucie MARIGNAC, éd. cit., p. 87-112: p. 91. J'emprunte la formule à Alain MOREAU, «Jason ou le héros évincé », dans Mythes et littérature, dir. par Pierre BRUnel, Paris, Presses de l'Université Paris-Sorbonne, 1994, p. 13-20.
} 
Je suis Jason, cellui qui le veaurre d'or conquesta en Colcos et qui journellement laboure en douleur, enrachiné en tristesse pour le deshonneur dont aucuns frapent ma gloire, moy imposans non avoir tenu ma promesse envers Medee, ce dont tu as leu la verité. Si te prie que tu faces un livre ou ceulz qui ma gloire quierent flappir puissent congnoistre leur indiscret jugement. Et a ce faire t'ay esleu affin que ton escripture presentes au pere des escripvains, c'est Philipe, par la grace de Dieu duc de Bourgoingne et de Brabant, VIe de ce nom, qui toute sa vie a esté nourry en histoires pour son singulier passetemps ${ }^{3}$.

Cette apparition de Jason au seuil du texte, par le biais d'un procédé métaleptique permettant au personnage de fiction de franchir le cadre de l'histoire et d'interpeller l'auteur, est censée renforcer et légitimer la nécessité de la réhabilitation: le projet entamé par l'auteur se présente comme une exigence venant de la part du héros lui-même. Le texte de Raoul se construit en outre comme une réplique aux voix qui s'élevaient dans l'entourage du duc contre le choix de l'Argonaute coupable d'infidélité et par conséquent indigne d'être considéré comme l'ancêtre symbolique de Philippe ${ }^{4}$. Jason ne trouvait pas sa place par exemple à «la table d'honneur» de dame Bonne Renommee, comme Gédéon, Alexandre, Arthur ou Charlemagne, dans le Songe de la Thoison d'Or de Michault Taillevent, écrit trois décennies auparavant. Dans la ballade qui clôt le songe, l'auteur met en opposition Gédéon dont la réputation était sans tache et Jason dont l'image était clairement dépréciée 5 .

\footnotetext{
${ }^{3}$ RAOUL LEFÈVRE, L'Histoire de Jason, ein Roman aus dem fünfzehnten Jahrhundert, édition critique par Gert PINKERNELL, Francfort-sur-le-Main, Athenäum Verlag, 1971, p. 125. Pour la traduction en français moderne, voir RAOUL LEFĖVRE, L'Histoire de Jason, traduit du moyen français, présenté et annoté par Lucie MARIGNAC, Récits d'amour et de chevalerie XII $-X V^{e}$ siècle, dir. par Danielle RÉGNIER-BOHLER, Paris, Éditions Robert Laffont, 2000, p. 10891217.

${ }^{4}$ Sur les enjeux de ce patronage mythique à la cour bourguignonne voir l'étude citée de Lucie MARIGNAC, p. 87-112.

${ }^{5}$ « Jason conquist, ce racontent pluseurs, / La thoison d'or par Medee s'amie / Dedens Colcos, mais pour estre plus seurs / Tant a Jason on ne s'areste mie / Qu'a Gedeon qui par œuvre saintie / Arousé eut son veaurre doucement / De rousee qui des saints cieulx descent / Dont fut
} 
Dans la première miniature du manuscrit 5067 de la Bibliothèque de l'Arsenal que le colophon désigne comme autographe et qui illustre la scène traditionnelle de présentation du livre achevé au prince, le chevalier Jason est figuré à droite, vêtu de vert et de rouge, un faucon au poing et le pied déchaussé, allusion à la fameuse prophétie du temple d'Apollo révélée à Pelleus l'usurpateur' ${ }^{6}$ Le choix de représenter Jason le faucon au poing n'est sans doute pas anodin ; oiseau noble, apanage des princes, le faucon est un indice du statut social du chevalier, un symbole hiérarchique qui rappelle la place exceptionnelle que Jason doit occuper dans l'imaginaire bourguignon. L'image fait également écho à l'épisode du songe prémonitoire du devin Mopsus qui présente Jason, futur conquérant de la Toison d'or, comme « un esprivier qui, querant sa proye, se bouta entre pluseurs oyseaulx terribles et d'estrange nature », avatars oniriques des taureaux et du dragon de l'île de Colchos, située aux confins du monde ${ }^{7}$.

\section{Vers la création d'une nouvelle figure royale : la reine Mirro}

L'intention de Raoul est clairement exposée, son roman transformera Jason d'amant ingrat et déloyal en un chevalier exemplaire, digne du destin que

depuis dignement celebree / Loenge a Dieu trestout premierement / Et aux bons gloire et haulte renommee.", Robert DeschaUX, Un poète bourguignon du XV siècle. Michault Taillevent (Édition et Étude), Genève, Droz, 1975, p. 82-83.

${ }^{6}$ Le colophon du manuscrit 5067 de la Bibliothèque de 1'Arsenal indique : «Escript de la main de l'acteur / Raoul Le Fevre, prebstre indigne ", RAOUL LEFÈVRE, L'Histoire de Jason, éd. cit., p. 240. Les miniatures de ce manuscrit sont attribuées à Loyset Liédet; cette première miniature se trouve au fol. $\mathrm{C}^{\mathrm{r}}$. La prophétie avait révélé à Pelleus, frère d'Oethés, que le futur roi de Mirmidoine serait le premier «homme qu'il rencontreroit ayant un pié deschaussié. », Idem, (7.2), p. 165. Voir également la quatrième miniature mettant en scène la rencontre de Pelleus avec Jason monosandalos, fol. $52^{\mathrm{V}}$.

${ }^{7}$ L' « oiseau sur la main signe la richesse et la haute position sociale du personnage mis en scène », cf. Corinne BECK et Elisabeth RÉMY, Le faucon, favori des princes, Paris, Gallimard, 1990, p. 58. «Plus que le chien, plus que le cheval, le faucon est l'animal préféré des chevaliers à partir du XII ${ }^{\mathrm{e}}$ siècle. C'est l'oiseau noble par excellence. Il est interdit aux vilains d'en posséder. ", $c f$. Michel Pastoureau et Gaston Duchet Suchaux, Le bestiaire médiéval. Dictionnaire historique et bibliographique, Paris, Le Léopard d'or, 2002, p. 68. Pour l'épisode mentionné du songe de Mopsus, voir RAOUL LEFĖVRE, L'Histoire de Jason, éd. cit., (15.5), p. 205. 
la cour de Bourgogne du $\mathrm{XV}^{\mathrm{e}}$ siècle lui réservait ${ }^{8}$. Une entreprise que la lentille contraignante de l'apologie ne rendait pas moins complexe. En effet, la réécriture du mythe impliquait principalement une reconsidération et implicitement une justification des histoires d'amour traditionnellement attribuées à l'Argonaute. La question épineuse de la fidélité amoureuse de Jason se situe au cœur de l'Histoire et elle n'est pas sans poser quelques difficultés au clerc dont l'attitude ironique fait parfois surface, le temps d'une glose (les prises de parole auctoriales par le biais des gloses sont le plus souvent ironiques ; un effet de distanciation se crée entre la matière mise en récit et sa position moralisatrice) :

« Moy? » respondy Jason, « que je fusse desleal a ma dame Mirro? J'ameroye mieux avoir la langue copee!» (Les gentilz hommes de maintenant feroient tantost ainsy que Jason. Toutesvoyez je ne conseille aux dames qu'elles s'y fient, car elles en pourroient estre habusees. $)^{9}$.

En d'autres mots, la réflexion de la glose mine la démonstration du récit; nuance subversive qui témoigne entre autres de la conscience de l'écart entre le monde des fables et le monde contemporain.

Le lecteur pourrait dans ce contexte se demander pourquoi l'auteur choisit d'inventer une nouvelle figure féminine, la reine Mirro, et d'ajouter une nouvelle aventure amoureuse au parcours de Jason, les séquences mythiques connues ne mentionnant, on le sait, que la reine des Lemniennes, Hypsiphyle, Médée et Créüse, la fille de Créon $^{10}$. Cette étude se propose de réfléchir sur la manière dont ce nouveau personnage fascinant se construit et s'intègre dans le

\footnotetext{
${ }^{8}$ « Rien ne saurait mieux illustrer cette alliance entre la Cour et la littérature, cette rencontre entre l'histoire et le mythe, que le succès de l'Ordre de la Toison d'or » $c f$. Joël BLANCHARD et Jean-Claude MÜHLETHALER, Écriture et pouvoir à l'aube des temps modernes, Paris, PUF, 2002 , p. 151.

${ }_{9}^{9}$ RaOUl LefÈvre, L'Histoire de Jason, éd. cit., (8.8), p. 172.

${ }^{10}$ Pour une présentation des cinq séquences du mythe antique de Médée et de Jason voir Duarte Mimoso-Ruiz, Médée antique et moderne. Aspects rituels et socio-politiques d'un mythe, Paris, Ophrys, 1982, p. 11-16.
} 
tissu narratif et dans le programme iconographique de certains manuscrits qui nous ont été transmis, ainsi que sur sa place dans la configuration du mythe argonautique ainsi reconstruit.

Trois récits composent l'Histoire de Jason : le récit du parcours de Jason désigné comme «matiere principal ${ }^{11}$ de l'ouvrage est entrecoupé par le récit de fondation de la cité de Jacointe, en Colchos, par le roi Appollo d'Arcade, ancêtre de Médée. Deux figures féminines dominent respectivement la première et la troisième section de l'Histoire : la reine Mirro et la princesse Médée. Le premier volet du texte qui s'achève avant l'expédition proprement dite en Colchide, figurée comme un dangereux archipel oriental, retrace les premiers faits d'armes de Jason (ses combats spectaculaires contre les Centaures aux noces de Pirithous, contre l'armée du roi d'Esclavonnie ${ }^{12}$ ou encore contre le géant Corfus afin de délivrer les habitants d'Olyferne) et met en scène cette première histoire amoureuse imaginée par Raoul ayant comme protagonistes Mirro et l'Argonaute. La figure de la reine d'Olyferne est placée au centre d'un véritable « roman d'amour conforme à la tradition médiévale », " invention romanesque [qui] vient donc au secours de la démonstration» auctoriale, comme le remarque Danielle Quéruel. ${ }^{13}$

Greffé en quelque sorte sur l'histoire mythique transmise par des sources connues en ce dernier siècle médiéval telles que le Roman de Troie de Benoît de Sainte-Maure qui s'ouvre sur la geste argonautique, l'Ovide moralisé, l'Histoire ancienne, l'Historia destructionis Troiae de Guido delle Colonne ou la Genealogia de Boccace, le récit de Jason à la cour de la reine Mirro se lit

\footnotetext{
${ }^{11}$ RAOUL LefÈVRE, L'Histoire de Jason, éd. cit., (13.1), p. 191.

${ }^{12}$ Les Esclavons symbolisent dans le texte de Raoul «la menace sarrasine », $c f$. Lucie MARIGNAC, trad. cit., n. 1, p. 1106.

${ }^{13}$ Danielle QuÉRUEL, «Jason, héros d'une biographie chevaleresque ? », Bien Dire et Bien Aprandre, $\mathrm{n}^{\circ}$ 20, «La biographie dans la littérature médiévale », textes réunis par Élisabeth GAUCHER et Aimé PetiT, Lille, 2002, p. 159-170 : p. 168 et respectivement p. 170.
} 
comme un écart stratégique et innovant ${ }^{14}$. Quel sens peut avoir pour le public bourguignon la figure de la reine Mirro ? Moins problématique que Médée la Colchidienne passionnée, fratricide et infanticide, plus réservée que la reine de Lemnos se glissant nue dans le lit de l'Argonaute qui refusait de lui céder, Mirro semble en effet incarner une figure courtoise idéale plus proche de la sensibilité du lecteur historique.

La création et l'insertion de cette figure royale édifiante à l'intérieur de la réécriture du mythe de Jason répondent sans conteste à un dessein idéologique. Plusieurs motivations semblent sous-tendre ce geste innovant et apparemment paradoxal : l'intention de subordonner l'exemplarité sans faille de Mirro au projet central de relecture de l'image de Jason, le désir de proposer une figure en rapport de concurrence et de complémentarité avec l'inquiétante Médée, et, sans doute, l'intention de se conformer au goût esthétique de la cour de Bourgogne, horizon d'attente précis pour lequel le clerc écrit et pour qui ces héros lointains de la fable grecque sont, pour reprendre la belle formule d'Estelle Doudet, « fantasmés plus que connus $»^{15}$.

Ainsi, le personnage de Mirro permet-il surtout de repenser en termes nouveaux la question centrale de la fidélité du héros et de remettre en cause sa contre-exemplarité. Le premier rôle de ce personnage nouveau qui investit la trame mythique est de tendre un miroir courtois et exemplaire à l'Argonaute. La jeune reine d'Olyferne, première femme à laquelle il promet amour et fidélité, fait qui lui confère une autorité certaine par rapport aux trois autres figures féminines du récit, est le symbole même de la dame noble et courtoise. Les paroles du roi d'Esclavonie qui esquissent un portrait idéalisé de la reine, contiennent en filigrane la signification de son nom :

\footnotetext{
${ }^{14}$ Pour une discussion des sources du roman, voir Gert PINKERNELl, éd.cit., p. 66-96 et Lucie MARIGNAC, art.cit., p. 102-106.

${ }^{15}$ Estelle DOUDET, «Le miroir de Jason: la Grèce ambiguë des écrivains bourguignons au $\mathrm{XV}^{\mathrm{e}}$ siècle ", La Grèce antique sous le regard du Moyen Age occidental. Actes du XV colloque international de l'Académie des Inscriptions et Belles Lettres, Nice, octobre 2004, Cahiers de la Villa Kérylos, ${ }^{\circ} 16,2005$, p. 175-193 : p. 176.
} 
O ma dame, par le jugement des hommes le vray ymage de sçavoir et a mon samblant le cler miroir de noblesse, oyez a voix de vostre servant par ma bouche implorant et requerant vostre pitié le plus treshumblement qu'il luy est [f. $12^{\mathrm{r}}$ ] possible. ${ }^{16}$

Modèle porteur d'une éthique et figure de savoir, ce sont les deux sens du mot miroir - exemplum et speculum - que le nom de Mirro dévoile. Avant de la peindre en amoureuse exemplaire, l'auteur insiste sur son statut de reine jeune mais «sage », décidée de combattre les envahisseurs esclavons afin de garder son honneur et de protéger son royaume ${ }^{17}$.

Remarquons également que Mirro apparaît en fin de compte comme la seule épouse que Jason ait vraiment choisie, sans subir de contrainte. Car s'il cède ultérieurement aux tentations d'Ysiphile lors du séjour des Argonautes à Lemnos, s'il s'éprend de Médée, ou encore s'il est sur le point d'épouser Creusa à Corinthe, ce n'est, souligne l'auteur, que contre son gré. Les enchantements de Médée et de sa nourrice en Colchide, qui ont pour effet immédiat la «mise en oubliance» de la belle Mirro, servent de prétexte pour disculper le comportement discourtois de l'inconstant Jason : «Qui de l'œil eslonge, du cuer se recule. Mirro est clere comme 1'or, mais Medee flamboye comme la pierre precieuse ${ }^{18}$, explique le chevalier ensorcelé à Mopsus. La mise en regard de ces deux figures féminines sur lesquelles se focalise le roman fait ressortir l'exemplarité sans faille de la première, même si Médée ne saurait être considérée pour autant comme un personnage entièrement négatif, comme l'a récemment démontré Maria Colombo Timelli ${ }^{19}$. Ses gestes les plus atroces, comme le dépècement de son propre frère ou les meurtres successifs de ses fils, cherchent une légitimation dans l'amour absolu qui la relie à l'Argonaute qu'elle

\footnotetext{
${ }^{16}$ RAOUl LefÈVRe, L'Histoire de Jason, éd. cit., (2.5), p. 134.

${ }^{17}$ Ibidem, (2.2-2.7), pp. 132-135. Mirro n'a que seize ans.

${ }^{18}$ Ibidem, (14.16), p. 200.

${ }^{19}$ Maria COLOMBO Timelli, art. cit., p. 80-88.
} 
désigne parfois comme véritable coupable ${ }^{20}$. À l'image complexe de la princesse de Colchos, à son altérité indéniable, le récit oppose l'image exemplaire et valorisante de la reine d'Olyferne, «fleur des dames", modèle de «bonté, beauté, sens et vertu $»^{21}$.

Si l'entrée en scène de la reine est antérieure aux séquences de l'aventure argonautique en Colchos, elle n'apparaît pas uniquement dans cette première section; en d'autres mots, le personnage n'est pas confiné à une présence épisodique. Le fil se renoue et le récit revient à Mirro après la scène de vengeance à Corinthe, où Médée avait déchiré et jeté dans les plats des convives les membres de son propre enfant; Jason la retrouve aux noces de Polus et de la fille d'Onestor. Accusé par la reine de n'être qu'un «malvais menteur » sans " foy ne loyaulté », échos probables des voix des détracteurs de la cour bourguignonne, Jason lui fait le récit de la « nuit mauditte» où il tomba amoureux de Médée et avoue son intention d'épouser la fille de Créon afin d'oublier la Colchidienne, mais omet de mentionner Hypsyphile ${ }^{22}$. Délivré des sortilèges de la Colchidienne grâce à la pierre "enclose » dans un anneau que Mirro lui offre, l'Argonaute renaît - le merveilleux est mis au service du projet éthique qui sous-tend le récit :

Jason prinst l'aneau et le mist en son doy, et lors tout a coup il oublia Medee et ne luy souvint de nulles dames que de la belle [f. $142^{\mathrm{v}}$ ] Mirro, ains perdy son triste maintien et recouvra nouvel estre $(\ldots)^{23}$

Déguisé en serviteur de Mirro, subterfuge visant à tromper Médée, Jason prend le nom de Sambor et épouse secrètement la reine, en présence d'un prêtre : autant d'éléments relevant de l'imaginaire romanesque qui ponctuent ce dernier récit. Une flèche de Patroclus tue Mirro, déclenchant l'errance de Jason à

\footnotetext{
${ }^{20}$ RAOUL LefÈVRe, L'Histoire de Jason, éd. cit., (20.3), p. 236-237.

${ }^{21}$ Ibidem, (4.8), p. 144.

${ }^{22}$ Ibidem, (19.1), p. 229-230.

${ }^{23}$ Ibidem, (19.2), p. 230.
} 
laquelle seule la réconciliation avec Médée mettra fin, dénouement inspiré probablement par Boccace ${ }^{24}$. L'intervention de la déesse Fortune n'est qu'un artifice permettant au roman de se clore par un retour aux deux héros de la mythologie grecque ${ }^{25}$.

\section{Reflets iconographiques du nouveau personnage royal}

Une confrontation des miniatures présentes dans les trois manuscrits conservés à la Bibliothèque nationale de France, enquête qui offre des éléments d'une réception historique du personnage inventé par Raoul, révèle des choix différents pour ce qui est de la représentation de Mirro ${ }^{26}$. Aucune des dix miniatures du manuscrit 5067 de la Bibliothèque de l'Arsenal qui se présente comme autographe ne figure la reine Mirro. Comment interpréter ce silence du programme iconographique attribué à Loyset Liédet ? Serait-il le signe de l'humilité de l'auteur envers toute une partie du texte qui est de son cru? Une seule des treize miniatures en grisailles du manuscrit BNF fr. 12570 met en scène Mirro : Jason en armure est agenouillé devant la reine ${ }^{27}$.

Le dernier manuscrit en revanche, BNF fr. 331, illustré par Liévin van Lathem et ayant appartenu à Louis de Bruges, seigneur de Gruuthuse, contient trois miniatures mettant en scène la reine : la première surprend son départ pour

${ }^{24}$ Genealogie deorum gentilium, Tutte le opere di Giovanni Boccaccio, a cura di Vittore BRANCA, Milano, Arnoldo Mondadori Editore, t. VII-VIII, 1998, a cura di Vittorio ZACCARIA, t. I, IV, xii, p. 394-398 et t. I, XIII, xxvi, p. 1306-1312 ; voir au sujet des sources possibles de cet épisode les explications de Gert PINKERNell, dans RaOUl LeFĖVRe, L'Histoire de Jason, éd. cit., p. 95 et les précisions de Maria COLOMBO TIMELLI, «Entre magie du savoir et magie de la parole: Médée dans l'Histoire de Jason de Raoul Lefèvre (vers 1460) », Actes du colloque "Magia, gelosia, vendetta. Il mito di Medea nelle lettere francesi », (Gargnano, 811 giugno 2005), Milan, Cisalpino, $\mathrm{n}^{\circ}$ 16, 2006, p. 71-88: p. 76-77. La réconciliation de Jason et Médée est selon Maria ColOMBo Timelli l'effet logique de l'effort constant de cette dernière d'expliquer et de justifier ses actes, même les plus cruels, et non la conséquence de son repentir final, art.cit., p. 87-88.

25 «Et Fortune, qui des choses mondaines trop se joue, amena Jason et la royne Mirro en la tour, ou ilz se mirent a une fenestre. ", RAOUL LEFÈVRE, L'Histoire de Jason, éd. cit., (21.2), p. 238.

${ }^{26}$ Pour la description des manuscrits et imprimés conservés, voir Gert PinKernell, éd. cit., p. 17-31.

${ }^{27}$ Paris, BNF, ms. fr. 12570 , fol. $36^{\mathrm{v}}$. 
Athènes afin de retrouver Jason (fig. 1), la deuxième réunit en une scène à trois personnages Médée, Jason et Mirro (fig. 2) et la troisième illustre la mort de la reine (fig. 3 ) $^{28}$. Ce manuscrit est postérieur à la mort de Philippe, « en son temps duc de Bourgoingne et de Brabant », comme le précise le prologue modifié, et est dédié au «tresredoubté seigneur monseigneur de la Gruthuse », fait chevalier de l'Ordre de la Toison d'or en $1461^{29}$. Scot McKendrick met en exergue le caractère exceptionnel de ce manuscrit qui se distingue des autres du cycle narratif de Jason par la complexité des cadres et des paysages illustrés, la richesse et la flamboyance des bordures ornées de grotesques et surtout par l'ampleur des scènes figurées dans les dix-huit enluminures de van Lathem, «the finest and most extensive sequence of images illustrating the story of Jason ${ }^{30}{ }^{30}$

Les trois miniatures de ce manuscrit figurant Mirro mettent en lumière des moments précis et symboliques du récit. La première, au folio $35^{\mathrm{v}}$, comprend trois scènes (voir figure 1, p. 93) : à l'arrière-plan Mirro seule dans sa chambre, en proie au désespoir suite au départ de Jason dont elle avait refusé, « la bouche close par honte », la requête amoureuse ${ }^{31}$, ensuite Mirro discutant avec sa fidèle servante, et, au premier plan, seule miniature offrant un magnifique portrait complet de la reine, Mirro à cheval, vêtue de bleu, couleur royale, "valorisée »

\footnotetext{
${ }^{28}$ Le ms. de Paris, BNF, fr. 331 contient dix-huit miniatures. Sur ce manuscrit et l'activité de LiÉVIN VAN LATHEM, voir Gert PinkeRnell, "Die handschrift BN ms. fr. 331 von Raoul Lefèvres "Histoire de Jason" und das Wirken des Miniaturisten Lievin van Lathem in Brügge ", Scriptorium, tome XXVII, 1973, p. 295-301 et la précieuse analyse donnée par Scot MCKENDRICK dans Illuminating the Renaissance. The Triumph of Flemish Manuscript Painting in Europe, éd. par Thomas KREN et Scot MCKENDRICK, The J. Paul Getty Museum, Los Angeles, 2003, p. 243-244 et p. 239.

${ }^{29}$ Paris, BNF, ms. fr. 331 , fol. $1^{\mathrm{r}}$ pour le prologue et fol. $163^{\mathrm{v}}$ pour la mention du nouveau dédicataire. Scot MCKENDRICK note au sujet de sa provenance que le manuscrit de Louis de Gruuthuse entre dans la possession de Louis XII comme l'atteste l'inventaire de Blois de 1518 (n. 115) et de 1544 (n. 1386) ; les marques bourguignonnes ont été repeintes, $c f$. Scot MCKENDRICK, Illuminating the Renaissance..., op.cit., p. 243. Je tiens à remercier Tania VAN HEMELRYCK pour ses suggestions précieuses concernant l'attribution et la possession de certains manuscrits bourguignons entrés ultérieurement dans les bibliothèques des rois de France.

${ }^{30}$ Ibidem, p. 243.

${ }^{31}$ RaOUl LefÈVRe, L'Histoire de Jason, éd. cit., (6.2), p. 153.
} 
et « valorisante $»^{32}$, s'apprêtant à quitter son royaume pour suivre Jason dans son expédition maritime vers la Colchide. L'accent est mis sans conteste sur la beauté de la reine située au centre de l'image; la fine couronne en or et les tresses qui dépassent sont deux éléments qui permettent de la distinguer de Médée dans l'ensemble des miniatures contenues dans ce manuscrit. La tonalité des couleurs est mise en relation par McKendrick avec une possible relecture sensible du miniaturiste soucieux de capter l'émotion de cet épisode poignant ${ }^{33}$.

La deuxième miniature, au folio $153^{\mathrm{v}}$, présente trois scènes dont l'une, à l'arrière plan, réunit en une même pièce les trois protagonistes : Jason, Mirro à droite et Médée à gauche (voir figure 2, p. 94). La scène qui a retenu l'attention du miniaturiste fait clairement référence à la rencontre imaginée par Raoul entre les trois personnages. En effet, Médée use de ses sortilèges et se transporte dans le château d'Olyferne (l'image la montre dans les bras d'un diable ailé) où se trouvent la reine et l'Argonaute :

Et Medee s'en ala en sa tente, puis estudia en sa science et en un moment se fist porter dedens Olyferne, en la propre sale ou Jason et Mirro estoient ensamble, parlans de leur affaire. Jason et Mirro eurent assez grande merveille de Medee quant soudainement en leur presence le veyrent. ${ }^{34}$

Le choix de peindre cette scène mettant en présence les trois acteurs de l'Histoire revêt une nuance symbolique : la reine Mirro, personnage inventé et rattaché au paradigme mythique argonautique récupéré par Raoul se situe au même plan que les héros que la tradition a pour ainsi dire canonisés.

Enfin, la troisième miniature, au folio $158^{\mathrm{v}}$, se focalise sur la scène tragique de la mort de la reine, à la fenêtre d'une tour de son château, le cou

\footnotetext{
32 Michel Pastoureau, Jésus chez le teinturier. Couleurs et teintures dans l'Occident médiéval, Paris, Le Léopard d'or, 1997, p. 133.

33 «Van Lathem's use of more muted color for the setting of the poignant scene where the lovesick young girl Mirro leaves home in poursuit of Jason reveals a sensitivity to the human emotions that lie behind the actions », remarque Scot MCKENDRICK, Illuminating the Renaissance..., op.cit., p. 243.

${ }^{34}$ RaOUl LefÈVRe, L'Histoire de Jason, éd. cit., (20.3), p. 236.
} 
transpercé par la flèche de Patroclus; cette illustration est la dernière du manuscrit (voir figure 3, p. 95).

L'insertion de miniatures représentant Mirro dans les manuscrits postérieurs au manuscrit 5067, et surtout dans celui illustré par van Lathem qui témoigne d'une reconsidération de certains choix iconographiques antérieurs, montre un intérêt indéniable pour l'héroïne et pourrait être interprétée comme un signe d'assimilation de cette nouvelle figure au récit mythologique réécrit qu'elle investit. Le roman de Raoul est, certes, l'histoire de Jason, à la fois emblème de l'Ordre institué par le duc de Bourgogne et reflet idéal de ce dernier, mais il est aussi l'histoire de l'entrée dans une configuration mythique remodelée d'un nouvel actant dont le parcours ne saurait demeurer un simple détour romanesque. Le récit de Mirro est plus qu'un «singulier passetemps » à l'usage des lecteurs de la cour bourguignonne: sous le voile de la fiction, les frontières du mythe semblent entrouvertes. 


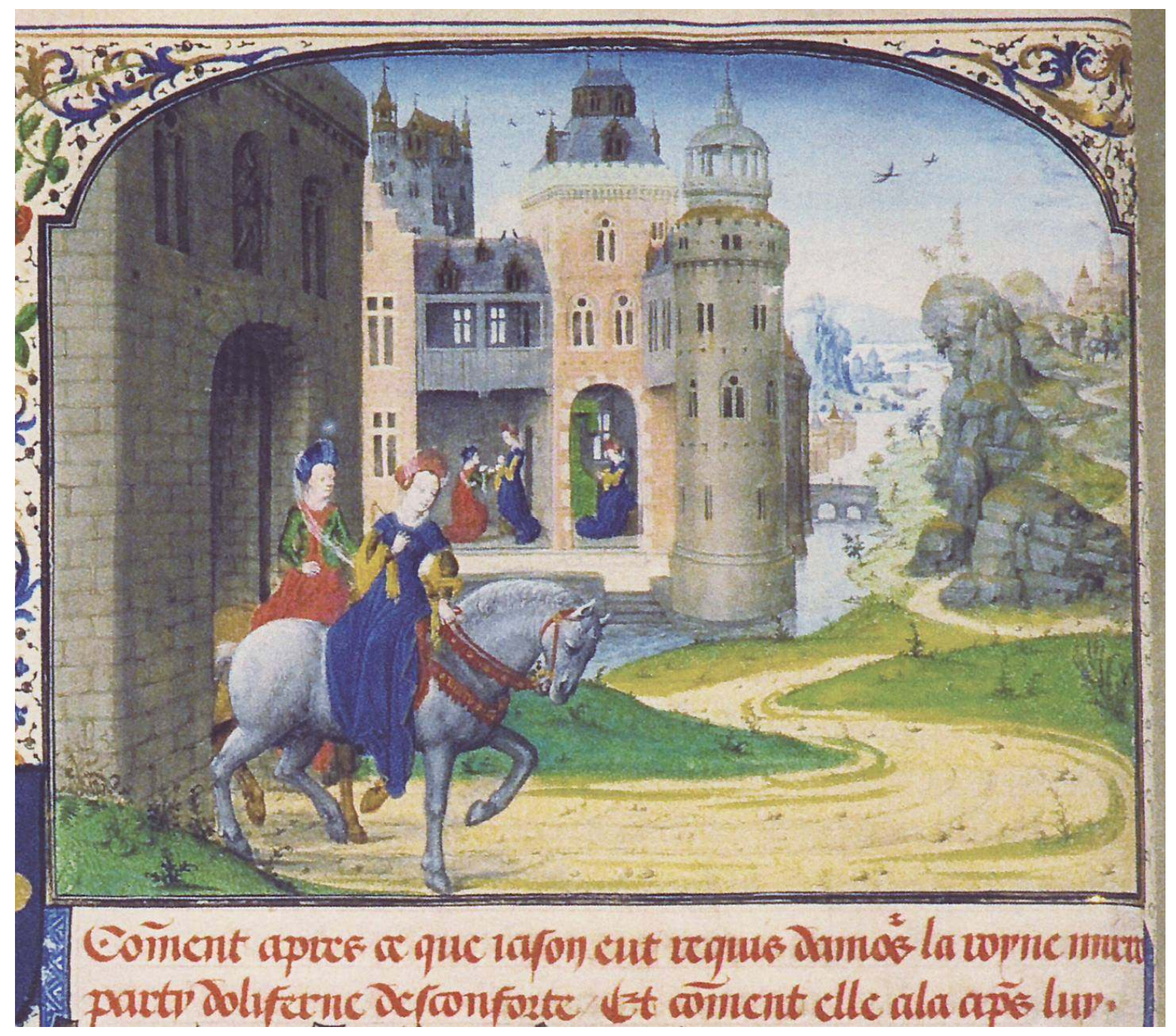

(fig. 1) 


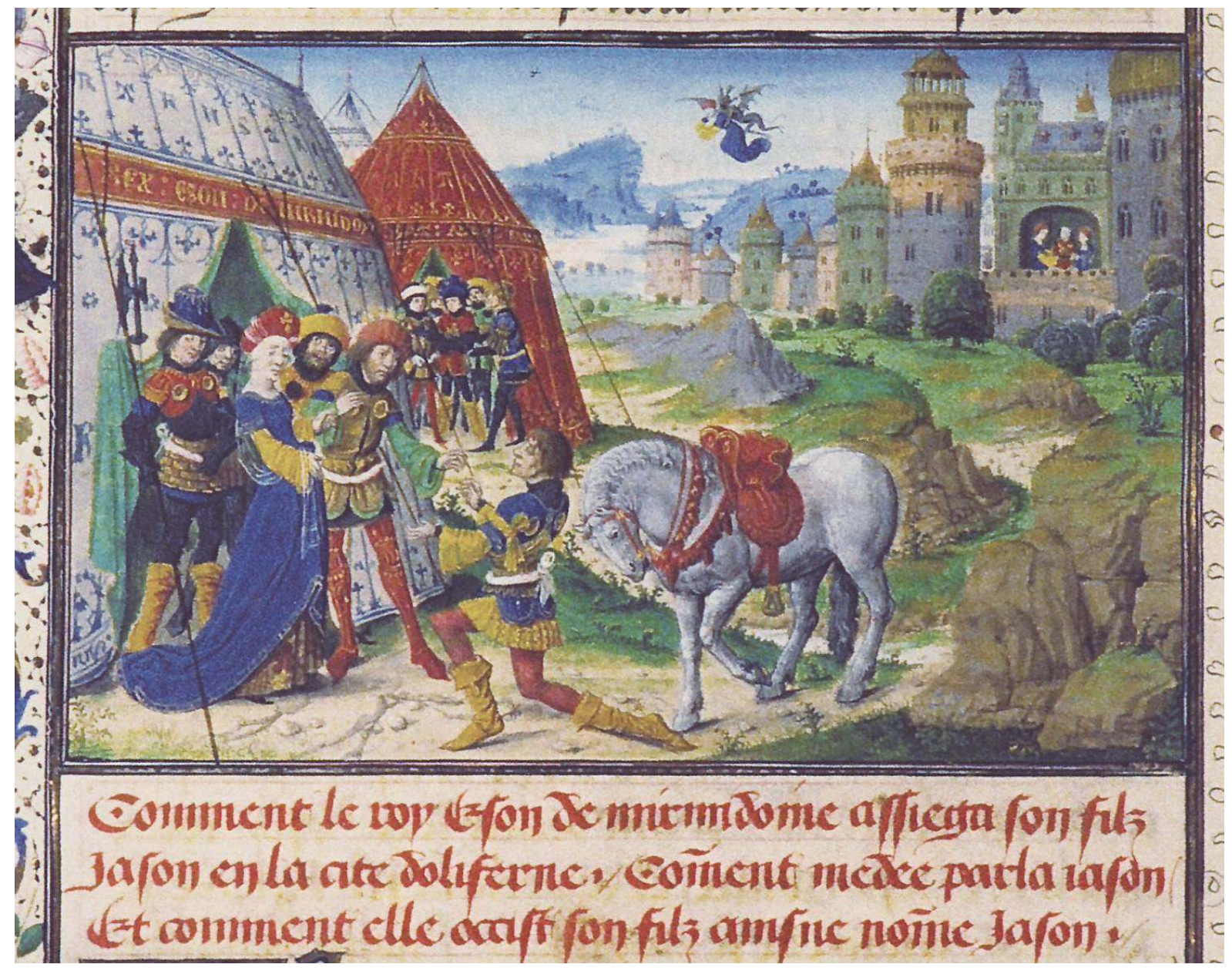

(fig. 2) 


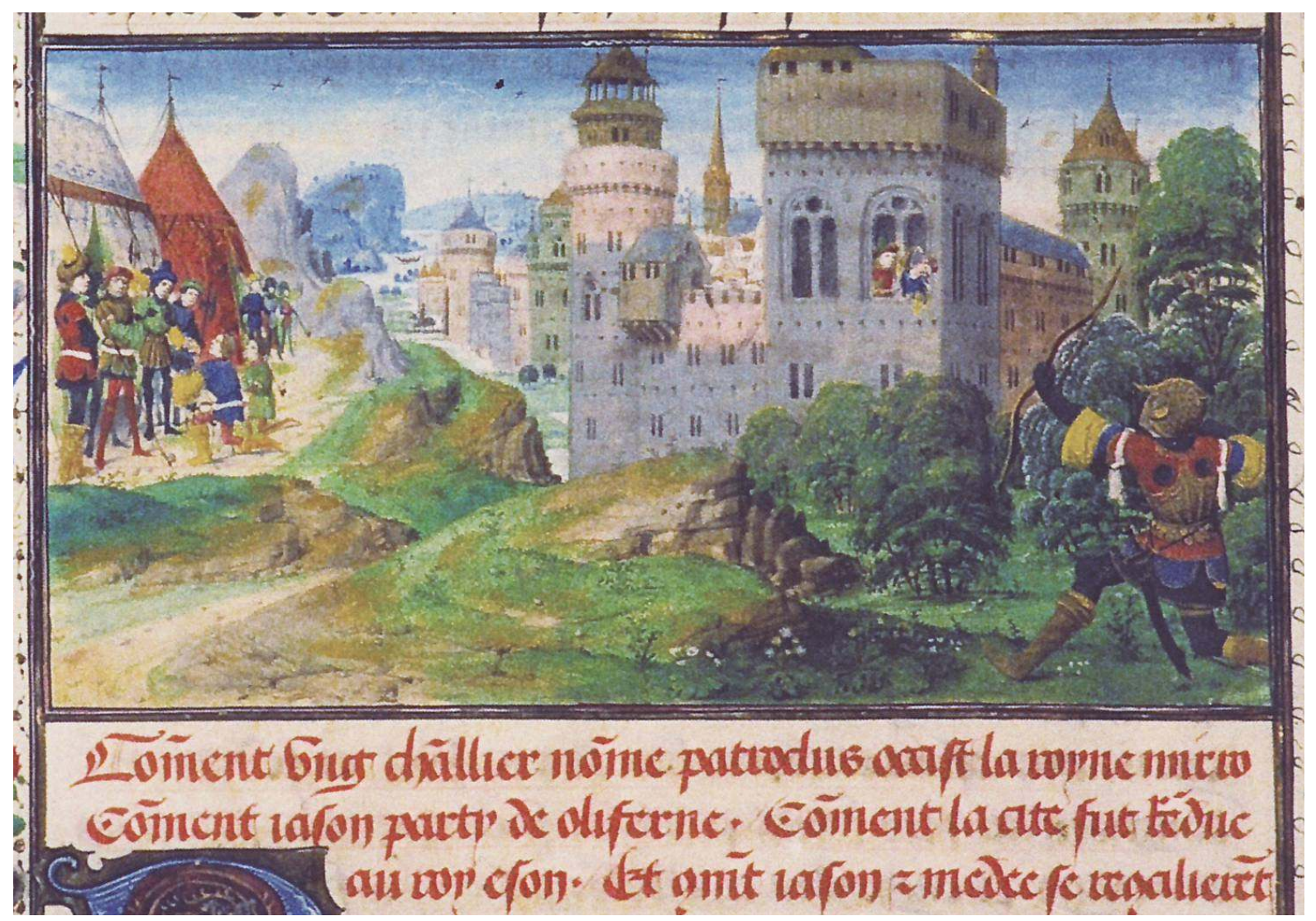

(fig. 3) 